\title{
Low threshold room-temperature lasing of CdS nanowires
}

\section{Citation}

Geburt, Sebastian, Andreas Thielmann, Robert Röder, Christian Borschel, Amanda McDonnell, Michael Kozlik, Julian Kühnel, Kristen A Sunter, Federico Capasso, and Carsten Ronning. 2012. "Low Threshold Room-Temperature Lasing of CdS Nanowires." Nanotechnology 23 (36): 365204. https://doi.org/10.1088/0957-4484/23/36/365204.

\section{Permanent link}

http://nrs.harvard.edu/urn-3:HUL.InstRepos:41371332

\section{Terms of Use}

This article was downloaded from Harvard University's DASH repository, WARNING: This file should NOT have been available for downloading from Harvard University's DASH repository.

\section{Share Your Story}

The Harvard community has made this article openly available.

Please share how this access benefits you. Submit a story.

Accessibility 


\title{
Low threshold room-temperature lasing of CdS nanowires
}

\author{
Sebastian Geburt ${ }^{1,3}$, Andreas Thielmann ${ }^{1,3}$, Robert Röder ${ }^{1}$, \\ Christian Borschel $^{1}$, Amanda McDonnell ${ }^{1}$, Michael Kozlik ${ }^{1}$, \\ Julian Kühnel $^{1}$, Kristen A Sunter ${ }^{2}$, Federico Capasso ${ }^{2}$ and \\ Carsten Ronning ${ }^{1}$ \\ ${ }^{1}$ Friedrich-Schiller-Universität Jena, Institut für Festkörperphysik, D-07743 Jena, Germany \\ ${ }^{2}$ Harvard University, School of Engineering and Applied Science, Cambridge, MA 02138, USA \\ E-mail: sebastian.geburt@uni-jena.de
}

Received 25 April 2012, in final form 27 June 2012

Published 21 August 2012

Online at stacks.iop.org/Nano/23/365204

\begin{abstract}
The synthesis of CdS nanostructures (bands, wires, irregular structures) was investigated by systematic variation of temperature and gas pressure, to deduce a comprehensive growth phase diagram. The high quality nanowires were further investigated and show stoichiometric composition of CdS as well as a single-crystalline lattice without any evidence of extended defects. The luminescence of individual nanowires at low excitation shows a strong near band edge emission at $2.41 \mathrm{eV}$ indicating a low point defect concentration. Sharp peaks evolve at higher laser power and finally dominate the luminescence spectrum. The power dependence of the spectrum clearly shows all the characteristics of amplified stimulated emission and lasing action in the nanowire cavity. A low threshold was determined as $10 \mathrm{~kW} \mathrm{~cm}^{-2}$ for lasing at room temperature with a slope efficiency of 5-10\% and a $Q$ factor of up to 1200 . The length and diameter relations necessary for lasing of individual nanowires was investigated.

S] Online supplementary data available from stacks.iop.org/Nano/23/365204/mmedia
\end{abstract}

\section{Introduction}

The miniaturization of light sources is one of the key tasks for the development of smaller optoelectronic devices with enhanced function and properties [1]. CdS nanowires are promising candidates as they offer efficient waveguiding [2], show light emission at electrical excitation [3], can achieve stimulated emission [4-6]; and even lasing at low temperatures [7] has been reported. The coupling of CdS nanowires to plasmonic substrates [8] resulted in the realization of a hybrid photonic plasmonic nanowire laser with extreme light confinement, but light amplification was only achieved at very high pumping due to the high optical losses of the plasmonic structure. For future integration of $\mathrm{CdS}$ nanowires into devices, two key points still have to be addressed: the investigation of the synthesis conditions for a cheap and reproducible production method for high quality nanowires and the systematic study of their emission properties in relation to the nanowire

3 These two authors contributed equally to this work. dimensions. Significant effort has already been devoted to the synthesis of CdS nanostructures ([9] and reference therein), but a comprehensive growth phase diagram has not yet been reported. Furthermore, most previous experiments on stimulated emission properties have been performed on nanowire ensembles [4-6], meaning that the properties of the individual nanostructure were lost in the superposition of the ensemble. Clear evidence for lasing action at room temperature and its relation to the morphology, as reported for $\mathrm{ZnO}$ nanowires [10, 11], is not yet available for individual CdS nanowires.

This study addresses the two key points: the systematic variation of the growth parameter for $\mathrm{CdS}$ nanostructures to achieve a cheap and reproducible routine for the synthesis of high quality nanowires, and the investigation of the individual optical properties with respect to the nanowire morphology.

\section{Experimental details}

Cadmium sulfide (CdS) nanostructures were synthesized by a chemical vapour deposition (CVD) process using the 

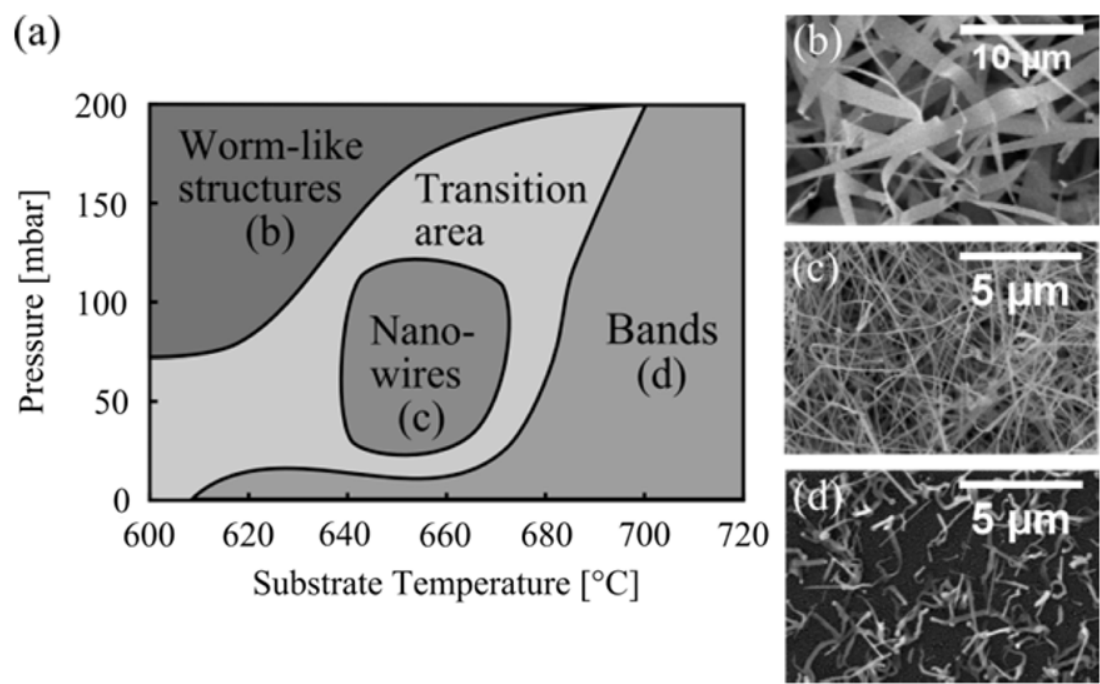

Figure 1. Several different morphologies of CdS nanostructures can be synthesized depending on the chosen growth conditions (temperature and gas pressure), as presented in the growth phase diagram (a). Representative SEM images display the CdS nanobands (b), nanowires (c) and irregular worm-like structures (d).

vapour-liquid-solid (VLS) mechanism [12]. CdS powder (99.99\% purity) was evaporated in a horizontal tube furnace at $800^{\circ} \mathrm{C}$ and transported by Ar gas at 5-200 mbar for $30 \mathrm{~min}$ towards the growth substrates ( $\mathrm{Si}$ chips sputter coated with $5 \mathrm{~nm} \mathrm{Au}$ as catalyst) located downstream within the furnace at temperatures between 720 and $600{ }^{\circ} \mathrm{C}$.

The morphology and crystal structure of the $\mathrm{CdS}$ nanostructures grown was characterized by scanning electron microscopy (SEM) and transmission electron microscopy (TEM), the stoichiometry was examined using energy dispersive $\mathrm{x}$-ray spectroscopy (EDX). Single nanowires were dispersed on to clean silicon substrates by the imprint technique for subsequent microphotoluminescence $(\mu \mathrm{PL})$ measurements performed with a home-built far-field epifluorescence microscope. The beam of either a $\mathrm{HeCd}$ laser $(325 \mathrm{~nm}, \mathrm{cw})$ or a frequency-tripled Nd:YAG laser $(355 \mathrm{~nm}, 10 \mathrm{~ns}$ pulses at $200 \mathrm{~Hz}$ ) was focused by a $40 \times$ refractive microscope objective $(\mathrm{NA}=0.7)$ to a spot size of $20 \mu \mathrm{m}^{2}$ diameter on the sample. The luminescence light was collected by the same objective, dispersed by a $500 \mathrm{~mm}$ monochromator (spectral resolution of $0.1 \mathrm{~nm}$ ) and detected by a nitrogen cooled charge-coupled device (CCD). For investigation of the emission from the nanowire facet end, nanowires partially suspended in air were optically pumped (Nd:YAG) perpendicular to the nanowire axis by a $40 \times$ refractive objective focused to a $75 \mu \mathrm{m}^{2}$ laser spot. The emission of the nanowire was collected 'head-on' by a $36 \times$ reflective objective in the direction of the nanowire axis at the air suspended end. The laser power was determined behind the microscope objective using an $\mathrm{Si}$ diode power meter and the excitation density was corrected for the laser focus diameter, the pulse length and the repetition rate. All $\mu \mathrm{PL}$ measurements were performed at room temperature.

\section{Results and discussion}

The substrate surface was covered with a wool-like yellow layer of CdS nanostructures after synthesis. The morphologies were systematically characterized by SEM and linked to the growth conditions (temperature and $\mathrm{Ar}$ gas pressure) to extract a clear phase diagram for VLS grown CdS nanostructures as presented in figure 1(a). No growth occurred above substrate temperatures of $720^{\circ} \mathrm{C}$ or below $600^{\circ} \mathrm{C}$, indicating that these temperatures are limits of the CVD process used $[9,13]$. Above $720^{\circ} \mathrm{C}$, the temperature is too high for a significant condensation of the source material; whereas below the lower limit, it is likely that the Au did not melt to form the droplets necessary for catalysing the nanostructure synthesis [14]. Within the growth temperature limits, several CdS nanostructure morphology types were found: band-like structures ('nanobands'; figure 1(b)) with a thickness around 100-300 nm, a width of $10 \mu \mathrm{m}$ on average and lengths of more than $100 \mu \mathrm{m}$ were synthesized at temperatures above $680^{\circ} \mathrm{C}$. The high growth temperatures and rich CdS vapour supply enable not only fast VLS growth along the band axis, but also slower lateral vapour-solid (VS) growth at the energetically favourable crystal sides leading to the formation of band-like structures [15-17]. At lower growth temperatures, the nanobands could only be found at low Ar pressures. Long and straight nanowires (figure 1(c)) were synthesized with diameters between 70 and $500 \mathrm{~nm}$ and lengths of more than $50 \mu \mathrm{m}$ at temperatures around $650^{\circ} \mathrm{C}$ and pressures of $50-100$ mbar. The intermediate temperature and the lower gas pressure, and therefore reduced CdS vapour supply, seem favourable for suppression of VS growth, enabling the synthesis of straight nanowires [13]. The remaining catalyst dots on top of many wires reveal growth by the VLS mechanism [12,9]. Shorter nanostructures with highly irregular morphology ('nanoworms'; figure 1(d)) occur at low temperatures and high pressures. In the transition area, a mixture of the morphologies described, e.g. tapered nanowires, was found between the conditions for nanowire and nanoband growth.

The morphology and stoichiometry of the straight nanowires was further examined by TEM and selective 

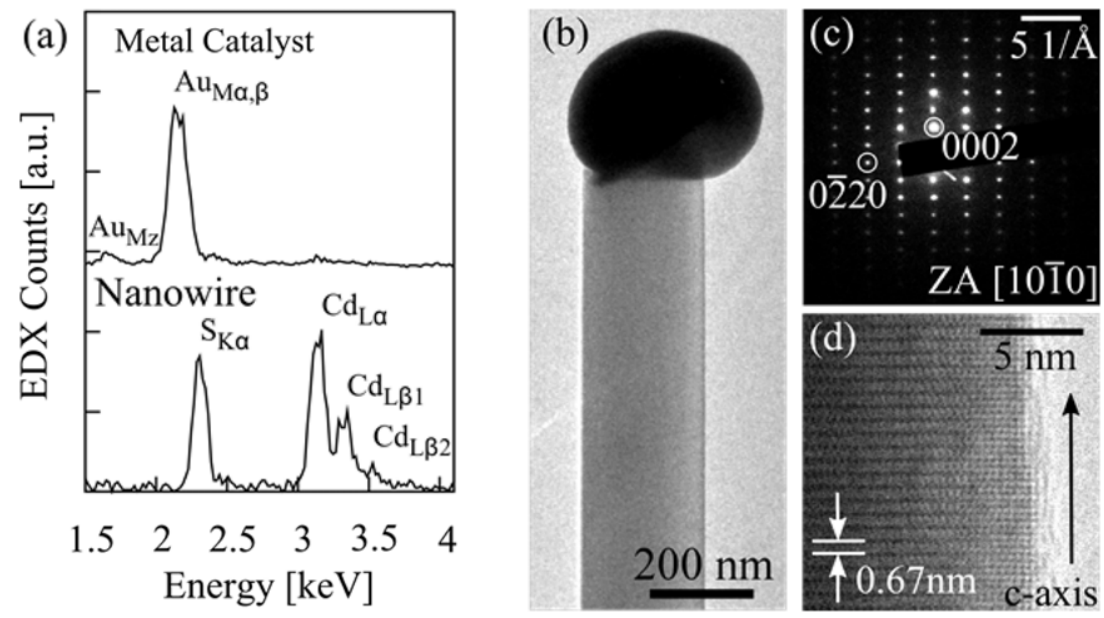

Figure 2. (a) EDX point analysis on a single nanowire in the TEM confirms the 1:1 stoichiometry of the CdS; whereas the remaining catalyst Au dot on top of the nanowire does not contain impurities. (b) The TEM image of a typical nanowire shows a very smooth surface without imperfections. (c) The SAED pattern of the nanowire body reveals the high quality lattice structure. The reflexes could be assigned to the spacing of the (0002) and (0220) planes (zone axis [1010]) of the wurtzite CdS crystal lattice, verifying growth of the nanowires along the $c$-axis. (d) The high resolution TEM image shows the crystal lattice; no extended defects such as stacking faults or dislocations could be observed.

area EDX measurements, as summarized in figure 2: the nanowires show a smooth and straight surface without extended imperfections (figure 2(b)). The remaining dot on top of the nanowire was identified as the Au catalyst, further confirming the VLS growth of the nanowires [12,9]. No impurities or residuals of the source material could be found in the Au dot (figure 2(a)). The composition of the nanowire is close to the stoichiometric ratio for $\mathrm{CdS}$ without any traces of the $\mathrm{Au}$ catalyst and other elements. The selected area diffraction pattern (figure 2(c)) of the nanowire body shows a large number of diffraction spots revealing a high quality crystal lattice. The assignment of the spots was performed by evaluation of the angles and distances to the central spot and matches very well to the spacing of the $(0002)$ and $(0 \overline{2} 20)$ denoted planes (zone axis [1010]) in wurtzite $\mathrm{CdS}$ (lattice parameters $a=4.16 \AA, c=6.76 \AA$ ) [18]. The growth of the nanowires could be clearly identified along the $c$-axis of the wurtzite $\mathrm{CdS}$ lattice (see supporting information figure $\mathrm{S} 1$ available at stacks.iop.org/Nano/23/365204/mmedia). The high resolution TEM image (figure 2(d)) shows the high quality single-crystalline lattice structure with a plane spacing of $0.67 \mathrm{~nm}$ along the $c$-axis in good agreement with the literature value. A very flat surface is observed (roughness < $1 \mathrm{~nm}$ ) and no hints for extended defects such as stacking faults or dislocations were found, revealing the high quality crystal structure of the $\mathrm{CdS}$ nanowires.

Microphotoluminescence measurements on single CdS nanowires (see supporting information figure S2 available at stacks.iop.org/Nano/23/365204/mmedia) at moderate cw excitation densities using the $\mathrm{HeCd}$ laser show a strong near band edge excitonic emission (NBE) centred at $2.41 \mathrm{eV}[19$, 20] as well as two weak defect-related emission bands at 2.08 and $1.67 \mathrm{eV}$ arising from carrier recombination at interstitial $\mathrm{Cd}$ atoms and sulfur vacancies [21, 22]. Power dependent measurements show a saturation of the defect band emission intensity around $0.1-1 \mathrm{~W} \mathrm{~cm}^{-2}$ due to the limited number of defects in the crystal while the NBE still increases linearly with excitation power. At higher excitation above $10 \mathrm{~W} \mathrm{~cm}^{-2}$, the NBE emission dominates the PL spectrum, confirming the low defect concentration inside the nanowires.

High resolution $\mu \mathrm{PL}$ spectra of the NBE under pulsed excitation by the Nd:YAG laser of a single nanowire (diameter $\sim 175 \mathrm{~nm}$, length $15 \mu \mathrm{m}$; see the upper inset of figure 3(b)) are displayed in figure 3(a). At a low excitation density, the broad NBE emission increases linearly with laser power, as shown in the integrated PL intensity versus pumping power plot in figure 3(b). The luminescence originates mainly from the position of the laser spot and waveguide effects lead to an additional emission from the nanowire ends (lower inset of figure 3(b)) [2, 23]. At excitation above $6 \mathrm{~kW} \mathrm{~cm}^{-2}$, sharp and equidistant peaks appear in the $\mu \mathrm{PL}$ spectra and show superlinear growth in intensity, which is interpreted as the onset of amplified spontaneous emission (ASE). Upon a further excitation increase above $11 \mathrm{~kW} \mathrm{~cm}^{-2}$, the sharp modes dominate the PL spectrum. The respective luminescence image now shows the strongest emission at the nanowire facet ends (see the inset of figure 3(b)). The data points follow a clear linear trend as the sharp peaks dominate the spectrum at high pumping power. The evaluated dependence includes all characteristics of a classical macroscopic laser system [24]. The emission properties and power dependence are similar to those for previously examined $\mathrm{ZnO}$ nanowire lasers [10]; therefore we conclude that the gain mechanisms of the $\mathrm{CdS}$ nanolaser follow the same principles [25]. The experimental values of the integrated PL intensity can be fitted with a model developed for multimode laser oscillators [26] using a fit parameter of $x_{0}=0.03$. This parameter determines the dependence of laser powers on pump intensity and the value is comparable to that for $\mathrm{ZnO}$ nanowire lasers [10]. The power dependence of $\mathrm{CdS}$ nanowires on plasmonic substrates can be fitted with smaller 
Photon Energy [eV]

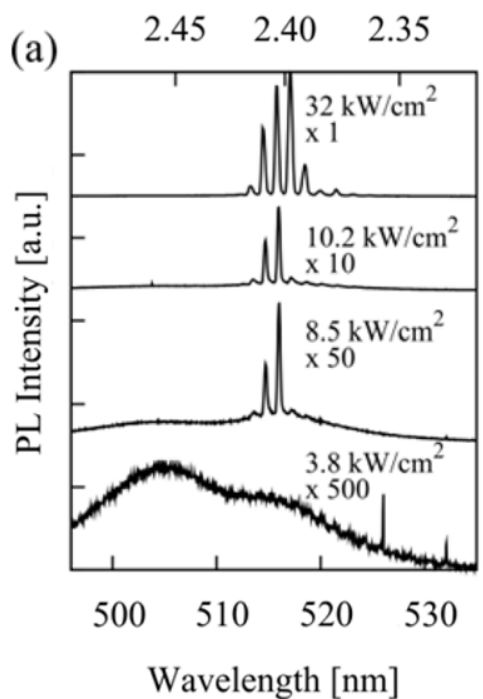

Gain-Loss Ratio

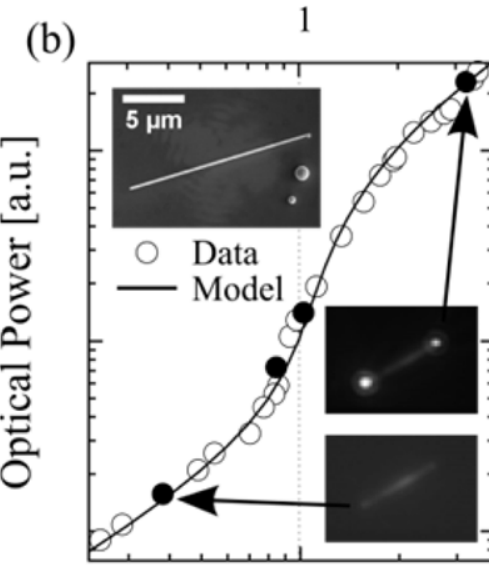

10

Pump Intensity $\left[\mathrm{kW} \mathrm{cm}^{-2}\right.$ ]

Figure 3. (a) $\mu \mathrm{PL}$ spectra of a single CdS nanowire (diameter $\sim 175 \mathrm{~nm}$, length $15 \mu \mathrm{m}$ ) at room temperature show the spontaneous emission of the near band edge emission at low excitation power. Above $6 \mathrm{~kW} \mathrm{~cm}^{-2}$, sharp peaks emerge indicating amplified spontaneous emission due to stimulated emission into the laser modes. At higher excitation, sharp, equidistant longitudinal laser modes dominate the emission spectra. (b) The s-shaped course of the experimental values in the integrated light intensity versus pump power plot clearly confirms lasing action of the CdS nanowire (SEM image of the respective nanowire with Au cap shown in the inset). The low threshold of $10 \mathrm{~kW} \mathrm{~cm}^{-2}$ was determined by fitting of the data with a model developed for multimode laser oscillators [26].

$x_{0}$ values, which is typical for hybrid systems with strong confinement [8].

From the fit of the experimental data, the threshold for lasing is obtained as the pump intensity at which the gain-loss ratio equals 1 . A lowest value of $10 \mathrm{~kW} \mathrm{~cm}^{-2}$ was evaluated for room-temperature lasing of the $\mathrm{CdS}$ nanowires. $\mathrm{ZnO}$ nanowires of comparable dimensions show lasing action at pump intensities which exceed the value determined for CdS by more than one order of magnitude $\left(\sim 300 \mathrm{~kW} \mathrm{~cm}^{-2}\right)$ [11]. Several factors contribute to this difference: in contrast to $\mathrm{ZnO}$ nanowires, most of the CdS nanowires investigated still had on one side the catalyst Au droplet attached-see the part (b) inset-leading to an enhanced reflection coefficient of the guided mode and reduced losses at the facet end as this is the dominant tone due to the short resonator length. A similar reflection enhancement was obtained for $\mathrm{ZnO}$ nanowires with Al-coated end facets [27]. A second possible explanation for the lower lasing threshold, compared to the $\mathrm{ZnO}$ nanowires case, is given by the longer emission wavelength of CdS: the inversion and lasing thresholds, necessary for gain and lasing, usually scale with the wavelength. In a 3D system, they are in fact inversely proportional to the wavelength squared [28], but for $1 \mathrm{D}$ systems this dependence is not that strong due to the reduced density of states of optical modes.

To gain further insights, the emission originating from the nanowire facet end was collected from a nanowire partially suspended in air in 'head-on' geometry (see the inset of figure 4(c)) without any scattering from the substrate, similar to what is reported in [11]. The nanowire output power was obtained by a calibration of the detection system using the reflection of a $532 \mathrm{~nm}$ laser of known power which simulated the nanowire laser emission; and subsequent conversion of the spectral data. Figure 4(a) shows the nanowire output power versus input pump power for a $17 \mu \mathrm{m}$ long CdS nanowire with a diameter of $\sim 700 \mathrm{~nm}$ (figure $4(\mathrm{~b})$ ). At an average excitation pump power below $10 \mathrm{nW}$, only spontaneous emission originates from the nanowire. The calculated ratio of nanowire light output to excitation laser input power increases from $\leq 1 \%$ for the spontaneous emission to a slope efficiency of $5-10 \%$ in the lasing regime, regarding only the emissions of the free standing nanowire end facet. This is also more than one order of magnitude higher than the value determined for a $\mathrm{ZnO}$ nanowire [11], which could further explain the lower lasing thresholds for $\mathrm{CdS}$ nanowires. The indirect measured 'head-on' data were confirmed by direct output power measurement using the Si diode power meter right behind the microscope objective (see the open triangles in figure 4(a)). The optical resonator can be characterized by the quality factor ( $Q$ factor) which is a common measure for the ratio of the stored to dissipated energy per oscillation cycle $[29,30]$. The value was calculated for each lasing mode by using $Q=\frac{\lambda_{0}}{\Delta \lambda}$, using the central mode wavelength $\lambda_{0}$ divided by the mode width ( $\Delta \lambda=$ FWHM). $Q$ factors of up to 1200 were obtained using the spectral data given in figure 4(c) at $120 \mathrm{nW}$ average input power, which is higher than the $Q$ factors observed in the literature [3, 31].

The length and diameter relations necessary for lasing action in $\mathrm{CdS}$ nanowires were obtained for measurements at $40 \mathrm{~kW} \mathrm{~cm}{ }^{-2}$ pump power and are displayed in figure 5 . It is obvious from the data that the CdS nanowires must have a critical length of about $7 \mu \mathrm{m}$. Below this value, the resonator cavity is too short to achieve sufficient gain to overcome the losses at the nanowire ends. A further limit was found for nanowires with diameters smaller than $170 \mathrm{~nm}$ : no lasing 


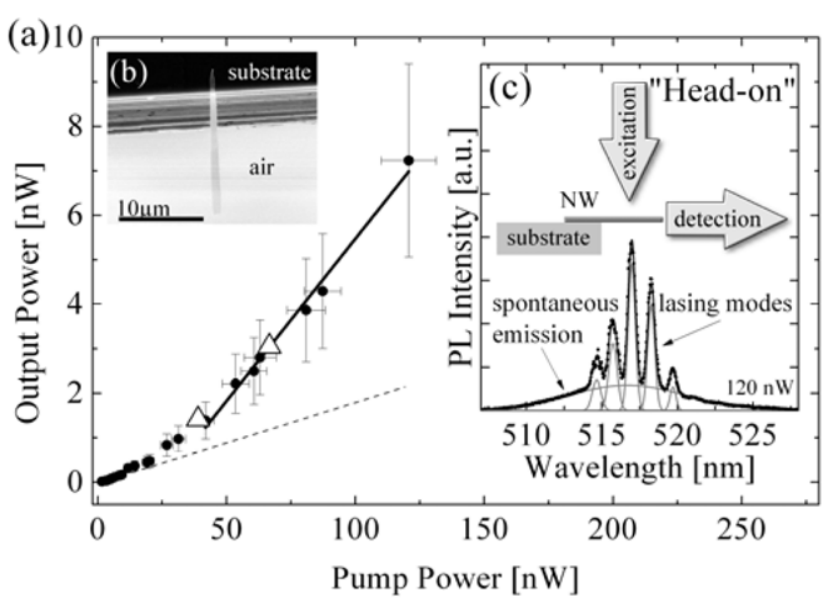

Figure 4. (a) Output power originating from a nanowire facet end versus average pump power measured in 'head-on' geometry shows typical lasing characteristics; a slope efficiency of $5-10 \%$ is estimated in the lasing regime. (b) SEM image of the CdS nanowire (diameter $\sim 700 \mathrm{~nm}$, length $17 \mu \mathrm{m}$ ) partially suspended in air. (c) The emission of the nanowire facet end at $120 \mathrm{nW}$ average pump power consists of sharp lasing modes superimposed to the broad spontaneous emission.

behaviour could be achieved below this value. Although some nanowires showed well defined waveguide modes on top of the strong ASE peak, the gain was insufficient to reach the lasing regime. The diameter threshold can be understood since the reflection coefficient at the nanowire ends and the waveguiding is related to the confinement of light in the nanowires [32]. As the nanowire diameter becomes smaller than a minimum diameter, light is no longer completely confined inside the nanowire, but propagates as an evanescent wave around the waveguide $[23,10]$. This leads to a strong increase in the losses in addition to the reduction of the reflection coefficient, suppressing the gain and therefore the lasing. As a rule of thumb, the minimum diameter can be estimated as $d_{\min } \approx \frac{\lambda_{0}}{n}$, where $\lambda_{0}$ defines the wavelength of the guided light $(515 \mathrm{~nm})$ and $n$ the index of refraction ( $n=2.76$ for CdS [33]). For CdS, the calculated minimum diameter is about $174 \mathrm{~nm}$, which is in good agreement with the experimentally determined value. Lasing at smaller diameters can be achieved [8], but the pumping threshold increases inversely to the decreasing diameter. The estimated diameter limit for $\mathrm{CdS}$ nanowires is slightly larger than the value for $\mathrm{ZnO}$ nanowires of $150 \mathrm{~nm}$ [11], which is due to the longer emission wavelength of $\mathrm{CdS}$.

The mode spacing of the sharp emission peaks depends on the nanowire length: shorter wires show only few resonant laser modes with a large spacing, while more resonant modes (with a smaller spacing) fit into the gain profile for longer wires. The mode spacing as a function of the inverse nanowire length evaluated is plotted in the inset of figure 5 . The data for all CdS nanowire lasers follow a linear relation, which is typical for Fabry-Pérot type cavities [24]. From the data of figure 5 , the dispersion for $\mathrm{CdS}$ at $517 \mathrm{~nm}$ is estimated as $\frac{\mathrm{d} n}{\mathrm{~d} \lambda}=-7.4 \pm 0.8 \mu \mathrm{m}^{-1}$, in good agreement with the literature value [33].

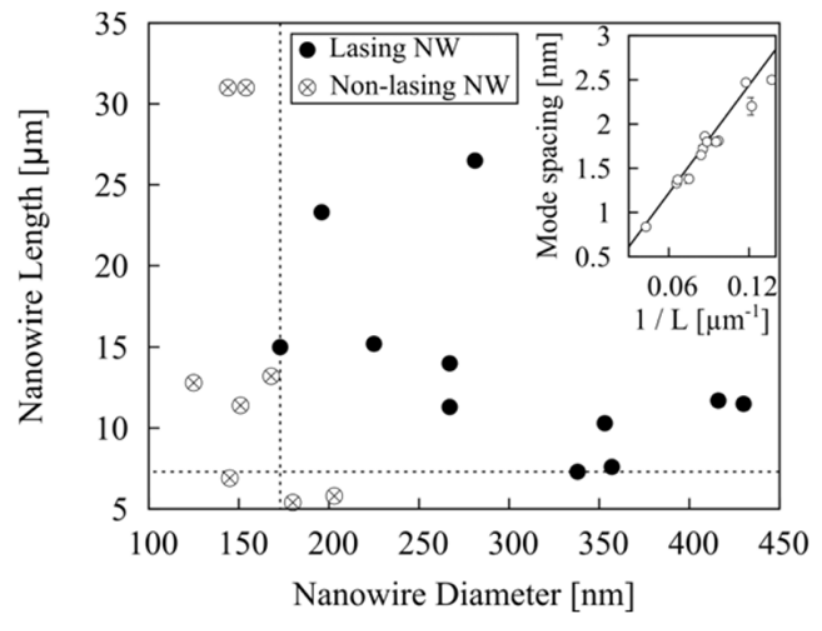

Figure 5. Measured length versus diameter relations required for lasing $(\bigcirc)$ and non-lasing $(\otimes) \mathrm{CdS}$ nanowires clearly show a minimum nanowire diameter of $170 \mathrm{~nm}$ required for lasing. Below nanowire lengths of $7 \mu \mathrm{m}$, no inversion can be reached due to the insufficient gain in one resonator cycle. The mode spacing evaluated follows a linear relation over the inverse nanowire length $1 / L$ confirming the Fabry-Pérot type resonator of the CdS nanowires (inset).

\section{Conclusion}

CdS nanostructures were synthesized via the VLS mechanism. The growth temperature and transport gas pressure were systematically varied and a phase diagram was evaluated for the morphologies of nanobands, nanoworms and nanowires found. EDX and TEM measurements show a stoichiometric composition of the high quality nanowires having a smooth surface and no extended crystal defects. Under optical excitation above the band edge, the nanowires emit a strong NBE emission and show a saturation of the defect emission at increased laser power. High pumping intensities lead to the appearance of sharp peaks on top of the broad emission, which were identified as lasing modes by the measured power dependence. The sharp modes dominate the emission spectrum above the low threshold of $10 \mathrm{~kW} \mathrm{~cm}^{-2}$ at room temperature. In the lasing regime, a slope efficiency of 5-10\% and $Q$ factors of up to 1200 were evaluated. The minimum diameter and length, required for lasing, were found to be $174 \mathrm{~nm}$ and $7 \mu \mathrm{m}$. The mode spacing shows all features of a Fabry-Pérot type cavity and the dispersion evaluated is in excellent agreement with the literature.

\section{Acknowledgments}

Financial support was granted by the DFG within the priority programme 1165 under contract No. Ro1198/7-3 and DAAD within the PPP exchange programme.

\section{References}

[1] Barrelet C, Greytak A and Lieber C 2004 Nanowire photonic circuit elements Nano Lett. 4 1981-5

[2] Pan A, Liu D, Liu R, Wang F, Zhu X and Zou B 2005 Optical waveguide through CdS nanoribbons Small 1 980-3 
[3] Duan X, Huang Y, Agarwal R and Lieber C M 2003 Single-nanowire electrically driven lasers Nature 421 241-5

[4] Liu Y K, Zapien J A, Geng C Y, Shan Y Y, Lee C S, Lifshitz Y and Lee S T 2004 High-quality CdS nanoribbons with lasing cavity Appl. Phys. Lett. 85 3241-3

[5] Pan A, Liu R, Yang Q, Zhu Y, Yang G, Zou B and Chen K 2005 Stimulated emissions in aligned CdS nanowires at room temperature J. Phys. Chem. B 109 24268-72

[6] Cao B, Jiang Y, Wang C, Wang W, Wang L, Niu M, Zhang W, $\mathrm{Li} \mathrm{Y}$ and Lee S 2007 Synthesis and lasing properties of highly ordered CdS nanowire arrays Adv. Funct. Mater. 17 1501-6

[7] Agarwal R, Barrelet C J and Lieber C M 2005 Lasing in single cadmium sulfide nanowire optical cavities Nano Lett. 5 917-20

[8] Oulton R F, Sorger V J, Zentgraf T, Ma R M, Gladden C, Dai L, Bartal G and Zhang X 2009 Plasmon lasers at deep subwavelength scale Nature 461 629-32

[9] Zhai T, Fang X, Li L, Bando Y and Golberg D 2010 One-dimensional $\mathrm{CdS}$ nanostructures: synthesis, properties, and applications Nanoscale 2 168-87

[10] Zimmler M A, Bao J, Capasso F, Müller S and Ronning C 2008 Laser action in nanowires: observation of the transition from amplified spontaneous emission to laser oscillation Appl. Phys. Lett. 93051101

[11] Zimmler M A, Capasso F, Müller S and Ronning C 2010 Optically pumped nanowire lasers: invited review Semicond. Sci. Technol. 25024001

[12] Wagner R S and Ellis W C 1964 Vapor-liquid-solid mechanism of single crystal growth Appl. Phys. Lett. 4 89-90

[13] Ge J and Li Y 2004 Selective atmospheric pressure chemical vapor deposition route to $\mathrm{CdS}$ arrays, nanowires, and nanocombs Adv. Funct. Mater. 14 157-62

[14] Castro T, Reifenberger R, Choi E and Andres R P 1990 Size-dependent melting temperature of individual nanometer-sized metallic clusters Phys. Rev. B 42 8548-56

[15] Kar S and Chaudhuri S 2006 Shape selective growth of CdS one-dimensional nanostructures by a thermal evaporation process J. Phys. Chem. B $1104542-7$

[16] Wang Y, Wang G, Yau M, To C and Ng D H 2005 Asymmetric growth on the polar surfaces of CdS ribbons Chem. Phys. Lett. 407 510-5
[17] Gao T and Wang T 2004 Catalyst-assisted vapor-liquid-solid growth of single-crystal CdS nanobelts and their luminescence properties J. Phys. Chem. B 108 20045-9

[18] Mandelung O 1996 Semiconductors_Basic Data (Berlin: Springer)

[19] Koch S W, Haug H, Schmieder G, Bohnert W and Klingshirn C 1978 Stimulated intrinsic recombination processes in II-VI compounds Physica Status Solidi b 89 431-40

[20] Shen G, Cho J H, Yoo J K, Yi G C and Lee C J 2005 Synthesis of single-crystal CdS microbelts using a modified thermal evaporation method and their photoluminescence $J$. Phys. Chem. B 109 9294-8

[21] Kulp B A and Kelley R H 1960 Displacement of the sulfur atom in CdS by electron bombardment J. Appl. Phys. 31 1057-61

[22] Aguilar-Hernández J et al 2003 Photoluminescence and structural properties of cadmium sulphide thin films grown by different techniques Semicond. Sci. Technol. 18 111-4

[23] Voss T, Svacha G, Mazur E, Müller S, Ronning C, Konjhodzic D and Marlow F 2007 High-order waveguide modes in $\mathrm{ZnO}$ nanowires Nano Lett. 7 3675-80

[24] Siegmann A 1986 Lasers (Sausalito: University Science Books)

[25] Shih T, Mazur E, Richters J P, Gutowski J and Voss T 2011 Ultrafast exciton dynamics in $\mathrm{ZnO}$ : excitonic versus electron-hole plasma lasing J. Appl. Phys. 109043504

[26] Casperson L W and Lunnam S D 1975 Gaussian modes in high loss laser resonators Appl. Opt. 14 1193-9

[27] Gruzintsev A, Emelchenko G, Redkin A, Volkov W, Yakimov E and Visimberga G 2010 Mode structure of laser emission from $\mathrm{ZnO}$ nanorods with one metal mirror Semiconductors 44 1235-40

[28] Meschede D 2010 Gerthsen Physik (Berlin: Springer)

[29] Paschotta R 2008 Encyclopedia of Laser Physics and Technology (Berlin: Wiley-VCH)

[30] Saleh B E A and Teich M C 2007 Fundamentals of Photonics (New York: Wiley-Interscience)

[31] Zhang Q, Shan X Y, Feng X, Wang C X, Wang Q Q, Jia J F and Xue Q K 2011 Modulating resonance modes and $Q$ value of a CdS nanowire cavity by single $\mathrm{Ag}$ nanoparticles Nano Lett. 11 4270-4

[32] Maslov A V and Ning C Z 2003 Reflection of guided modes in a semiconductor nanowire laser Appl. Phys. Lett. 83 1237-9

[33] Bass M 2010 Handbook of Optics (New York: McGraw-Hill) 